\title{
REVIEW OF INCLUSION OF MACHINE LEARNING TECHNIQUES IN MEDICINE FIELD
}

\author{
Samaksh Khandelwal \\ Student, Seedling Modern Public School, Udaipur, India
}

\begin{abstract}
The field of medicine is seeing indomitable changes and development with the inclusion of machine learning techniques and artificial intelligence. These inclusive systems are improving the overall efficiency and accuracy in every facet of medicine: Prognosis, diagnosis, and treatment are all positively impacted. The understanding of machine learning, its nuances, and techniques will be transforming the field of medicine in the coming years. This paper aims to learn and review the effect of machine learning and its inclusion in the medical field spanning pathology, radiology, mental health, and dentistry. There is an indepth understanding of how these inclusive systems could address the problems faced by patients and physicians leading to effective diagnosis and treatment. The research methodology is the systematic literature review of articles with the cluster of keywords identified as the central objective.
\end{abstract}

\section{KEYWORDS}

Machine learning, healthcare, artificial intelligence, diagnosis, pathology, radiology, dentistry, mental health

\section{INTRODUCTION}

The term 'machine learning' was coined by Arthur Samuel(2000) in the IBM Journal of Research and Development as "a field of study that gives computers the ability to learn without being explicitly programmed". It is the capability that devices hold to learn from their experiences and not with the help of solely programming and coding. The most common example of this phenomenon is the 'related search' that pops up on your screen based on your previous purchases or search history.

Artificial intelligence, the term first coined by John McCarthy at a conference in Dartmouth(1956), is explained as "the science and engineering of making intelligent machines"(Society of the study of artificial intelligence and simulation behavior,
2018). Machine learning, which is a subset of artificial intelligence, is the study of providing machines with human-like behavior and the ability to produce their own programs and algorithms with minimum to no human intervention. The automated tasks that require intrinsic human intelligence can also be fulfilled by machine learning; hence the field of machine learning and artificial intelligence is looked up to in every field including business, medicine, commuting, corporate sectors and so much more.

Machine learning and artificial learning have brought in an improved experience in the field of medicine by providing aid in improving the health of populations through faster and efficient prognosis, diagnosis, and treatment. This in turn reduces the cost of healthcare and improves the life of healthcare providers. Artificial intelligence has delivered a positive impact on the interpretation of digital imaging thereby helping in fields such as radiology and oncology. Detecting lesions with high efficiency in pathology is one of the applications of AI which displays its beneficial effect. Dentistry has also been seen using artificial intelligence and machine learning for dental restorations and prosthodontics making the entire process convenient with lesser room for error.

The healthcare industry is upholding a flood of medical information and records as big data and now with the help of ML techniques and AI, that data can be accessed in a streamlined way for better understanding and apprehension.. This helps doctors and healthcare providers in improving the quality of healthcare provided to their patients with more affinity for success.

Radiologists on the other hand are provided with tools and techniques that help them in assessing deep imaging more efficiently and effectively.

A research firm called Frost and Sullivan has estimated that artificial intelligence and machine learning inclusion in healthcare has the potential to increase patient outcomes by $10 \%$ and also reduce overall treatment costs by $50 \%$. The medical fraternity and potential for further betterment is what is looked up for in Artificial intelligence and 


\section{International Journal of Engineering Applied Sciences and Technology, 2021 \\ Vol. 6, Issue 5, ISSN No. 2455-2143, Pages 220-225 \\ Published Online September 2021 in IJEAST (http://www.ijeast.com)}

machine learning techniques which are slowly but steadily improving the field of medicine in detection, diagnosis, drug manufacturing, and in diagnostic radiology. Each and every field like pathology, ophthalmology is seeing great improvements with the integration of these systems. However these also come with certain limitations that we have discussed in the later portion of the paper. The practical inconvenience in getting these into clinical practice is on high scales, but the future of medicine looks bright with this integration as there will be more convenience, ease, time efficiency and smoothness.

This paper is, henceforth, organized as follows. The literature survey encompasses the survey regarding research area; a discussion on how machine learning techniques and artificial intelligence could change the future of medicine in the fields of dentistry; mental health; radiology; oncology, and pathology. This is followed by an overall review of the inclusion of machine learning techniques in the field of medicine with conclusion at the end.

\section{METHODOLOGY}

The survey methodology of systematic literature review has been used. The articles reviewed in this paper have been obtained through different scholarly websites like research gate, PubMed, and various other related textbooks. The keywords that helped in retrieving the selected articles were machine learning/ML, artificial intelligence/AI, healthcare, diagnosis, medicine, radiology, oncology, pathology, mental health. The articles which showed a relation between artificial intelligence, machine learning, and medicine were considered. They included both qualitative and quantitative ones. The qualitative articles provided a deep understanding of the semantics and gave insights related to the topic whereas the quantitative ones provided patterns in accordance with the facts of the topic.

\section{LITERATURE SURVEY}

(Patel et al.,2009) presented his paper titled " The coming of age of artificial intelligence in medicine". He reflects and discusses how artificial intelligence and machine learning integration has helped the medical fraternity and how it could change in future times. He opines that machine learning and artificial intelligence inclusive systems can be used in clinical practice for more research and to facilitate better functioning of physicians and healthcare providers. He also mentioned that as medical fields hold a great deal of data, it would be of immense help to physicians and doctors if the systems could find a smooth and streamlined channel for the retrieval of such sensitive information.

(Kralj and Kuka, 1998) mentioned that one of the very important applications of computer systems is in the field of medical diagnostic reasoning. The generation and prediction of patient data can be done effectively within this framework. Machine learning provides tools that can effectively read through different characteristics of medical databases helping in creating and improving medical decision making processes.

The impact of machine learning and artificial intelligence in the radiology field was discussed by (King $J r, 2018$ ). He explained how the field of radiology has already improved a lot with the advent of scanning technologies like ultrasound, computed tomography(CT), positron emission technology(PET), and magnetic resonance imaging(MRI). He also believes that the next stride of artificial intelligence would be the identification and isolation of pathological lesions thereby helping the radiologists come to conclusions faster hence making the process time efficient. Even in the field of oncology, diagnostic imaging computer systems help to identify any lesions in the samples or skin tissues in lesser time thus making the diagnosis faster and more effective.

There is an immense study and discussion on the topic of reviewing these images by an artificial intelligence tool at first and then by radiologists thus increasing efficiency and functionality. This AI tool with machine learning techniques can help in analyzing and creating a pattern sooner than a human can process thereby providing a faster diagnosis and treatment plan which could improve the life expectancy of the concerned patient.

In the field of mental health and psychiatry, artificial systems and machine learning techniques are used for emergency psychiatric state prediction. This helps in understanding the patient's state better and give emergency treatment at particular mental states. Using biosensors, it is extremely challenging to predict any change of state but the psychometric features of the patient along with their history- help the model recognize and observe any change of state. With this prediction, an algorithm can be proposed and doctors may be well prepared to provide better treatment against any psychiatric risks in future.

Roach 2017 has explained how artificial intelligence helps in the field of ophthalmology. The retina could be widely seen through deep learning AI and predictions of diabetic retinopathy 


\section{International Journal of Engineering Applied Sciences and Technology, 2021 Vol. 6, Issue 5, ISSN No. 2455-2143, Pages 220-225 \\ Published Online September 2021 in IJEAST (http://www.ijeast.com)}

are observed and made note of. The systems inclusive of machine learning techniques could very easily detect these eye problems and hence provide the right diagnosis and treatment for the patient. Machine learning has been seen to be involved in various fields of medicine like disease identification; diagnosis; personalized treatment; behavior modification;drug discovery; manufacturing;clinical trial research; radiotherapy;radiology;smart medical health reports; and epidemic outbreak prediction. Prediction and detection have been an application field for artificial intelligence and its subset machine learning.

Norman 2018, in his article" your future doctor may not be a human", mentioned how machines could overtake the roles of doctors and why machine learning inclusion is not for putting human minds against the machines. He mentioned how an enormous load of medical databases can be very easily processed by the machines. The point he mentions is to have an equally divided function so that the overall healthcare could be improved for the patient within a lesser time frame and with more effectiveness. Both the human minds and the artificial tools could co exist together working towards one directed goal which will benefit the entire healthcare industry.

A medical futurist called Mesko(2017) mentioned that artificial intelligence, along with machine learning, is the stethoscope of the 21 st century. He compared both of these facets and mentioned that when the stethoscope was first introduced, it took quite some time for it to get accepted among the medical fraternity. Similar will be the case of machine learning inclusive systems. He mentioned the importance of teaching the healthcare providers how to make better use of these systems to improve the overall health care system and quality of service offered to the patients. He pointed out companies like IBM and asked them to take up this opportunity as a slate to try and incorporate the use of such systems by the medical fraternity for it to get totally accepted.

Zaidi 2018 in his article "the 3 most important applications of AI in healthcare" mentioned the importance of artificial intelligence inclusive systems for surgeries requiring extreme precision. The da Vinci model, which has robotic limbs and assists surgeries nowadays with its three dimensional magnification, has been quite on the stir and it is mentioned that the use of this can help surgeons get the work done more efficiently with less stress. Furthermore, the incorporation of AI tools in administration helps in getting all the other works like mentioning prescriptions, tests orders and other chart notes to be taken care of.

Houssami 2017, in the article "artificial intelligence for breast cancer: Opportunity or hype", mentioned the importance of machine learning techniques and inclusive systems in mammograms which help in early breast cancer detection. It is also mentioned that radiologists are sometimes not able to see through the images with their naked eye and this leads to false positives or negatives which are not desirable. With the help of artificial intelligence and machine learning, the computer systems can see through the pixelated images for any minute changes and come to a concrete diagnosis thus helping the patient and healthcare system.

\section{MACHINE LEARNING, ARTIFICIAL INTELLIGENCE AND THE FUTURE OF MEDICINE}

The inclusion of machine learning techniques and artificial tools in medicine and healthcare has revolutionized the health field over the past few decades. The amount of healthcare data that is collected can now be accessed with more ease and convenience. Various monitors with multiple health parameters can help the patient keep a tab on their health and also help the doctor understand the progression course of the patient's health. Even though the advancements in this field are still pretty slow, in the long run, it will make a positive impact on the medical field. Most of the fields in medicine are seeing a new trend with these tools. Here we are going to discuss its impact and future of some medical specializations like radiology, dentistry, pathology, mental health, oncology, ophthalmology. This will give an overall idea of the potential of these systems in healthcare.

\section{Radiology}

The field of radiology is used to detect any abnormalities in the patient. With an extreme load of data and images together, it takes hours for the human eyes and radiologists to come to a conclusion. This is when the artificial intelligence tools along with machine learning techniques come in handy. The immense amount of data can be easily processed with an eye for any pathological abnormality by these systems. This integration of machine learning techniques can transform the field of radiology for the better. The radiologists along with these systems will be able to come to conclusions and diagnosis in less time with the highest possible accuracy without any room for error. The algorithms that the system integrates will be able to interpret the pathology of the image 


\section{International Journal of Engineering Applied Sciences and Technology, 2021 Vol. 6, Issue 5, ISSN No. 2455-2143, Pages 220-225 \\ Published Online September 2021 in IJEAST (http://www.ijeast.com)}

and radiologists along with other physicians can follow pathways and plans for treatment for further recuperation of the patient. Lakhani and Sundaram 2017 through their research study of using algorithms for the diagnosis of tuberculosis gave in a whopping $96 \%$ in its accuracy levels. This diagnosis was made after intricate learning of over a thousand $\mathrm{X}$ rays and this accuracy level is comparable to humans indicating that their efficiency is at par with human minds. Similarly, even breast cancer lesions were tested with deep learning algorithms of artificial intelligence and machine learning techniques and the results gave performance that was at par with the radiologist's level and capability. These collaborations between the radiologist and the systems bring in faster image detection and diagnosis thus giving better results for both the patients and the healthcare system.

\section{Pathology}

Pathology is the rendering of diagnosis through a bio specimen by a very experienced pathologist. With the increase of sets and subsets of diseases coupled with the fact that fewer students pursue this career, the field has been facing a lot more challenges. This increases the work of existing pathologists as a whole. This is when the deep learning approach of machine learning and artificial intelligence tools come in handy. The efficiency of these systems has been tested time and again. There is one research study done by google where the breast cancer lymph nodes were used and the accuracy was at par with human counterparts which led to pathologists making it a newer addition to the topic of application of AI in this field. The element of fatigue and exhaustion is not applicable for the machine and this is one major advantage of these systems as opposed to human pathologists. The level of accuracy will also be the same or even higher. Human pathologists might be overwhelmed with unlimited biomarkers present whereas machines with their ML techniques could process through any genomics data and continue to do so until they are shut down. As mentioned earlier, the inclusion of machine learning techniques helps in detection as well as prediction. There have been cases wherein the systems have predicted how long the kidney of a person with real biopsies would be functioning making it easier to plan and treat the said patient. It is very clear that artificial intelligence systems have the potential to augment any clinical decision-making for healthcare providers.
The use of machine learning techniques and artificial intelligence is present in the detection of retinal diseases and these techniques are set to revolutionize the field of vision care. The field already has an AI based tool to easily detect diabetic retinopathy disease quickly. The different amounts of retinal fluids are also analyzed by the machine with utmost precision and this is why there is high use of deep learning techniques in retinal image analysis. They are also used to diagnose retinal disease among pediatric patients. The use of these tools and machines have made diagnosis in the sensitive areas of the eye and retina easier than ever. It has become imperative that ophthalmologists learn how to use machine learning techniques and tools for their betterment in the field.

\section{Mental health}

The field of mental health deals with the mood and behaviors of people which are extremely hard to predict through present biosensors. Looking at previously discussed sections, it is very clear that the artificial intelligence and machine learning tools can also very easily predict the mental behavior of patients thus helping in providing the right treatment. Even scales like the Hamilton Anxiety scale, Beck Depression scale are insufficient to predict the behavior and mind of patients effectively, and hence AI tools can come in handy at such times for emergency psychiatric evaluation and prediction. For example, Suicidal tendencies can be managed by an AI tool which is wearable and is non invasive.

\section{Dentistry}

The field of Dentistry has a lot of laboratory work which involves building up prostheses for teeth. Thus rapid prototyping and other machine learning techniques are now employed in the field of dentistry. The fabrication of dental prostheses is usually very time consuming and an extremely detailed work which if conventionally done has errors associated with it. The manual errors by the dentists can now be avoided by RP techniques and use of computer systems. The steps that a dentist does are reduced therefore a lot of time is saved in the reconstruction of these dental models. The level of accuracy is also high and efficient. For different applications and needs, you can use these machine learning techniques accordingly for ease and convenience. In the field of Prosthodontics, the system will be very well used in the coming future enabling dentists to only make the molds while the manufacturing is done by the systems.

\section{Ophthalmology}




\section{International Journal of Engineering Applied Sciences and Technology, 2021 Vol. 6, Issue 5, ISSN No. 2455-2143, Pages 220-225 \\ Published Online September 2021 in IJEAST (http://www.ijeast.com)}

\section{OVERALL REVIEW OF INCLUSION OF MACHINE LEARNING \\ TECHNIQUES IN MEDICINE FIELD}

Machine learning and artificial intelligence have been quite the talk of the town in healthcare and the medical field. In such a manpower driven industry like medicine, AI tools are heartily welcomed. Through the research paper, it is seen that the applications of AI and ML in multiple fields like ophthalmology, pathology, mental health, radiology, and dentistry have brought huge help for the healthcare personnels and will continue to bring in more developments in the future.

Artificial intelligence and machine learning techniques will remove a lot of burden from the lives of healthcare providers even though these techniques are not easily taken in by the medical fraternity. The ease and convenience of AI involved in the process along with high accuracy and quick diagnosis are what make the system acceptable. In coming times, it would become imperative that the application and knowledge of the artificial intelligence tools are learned by all of the healthcare providers for the progress of the entire field.

With so many advantages of applications of artificial intelligence and machine learning techniques in the medical field, there are also some limitations when it comes to their integration in clinical practice. There has been a lot of research and the potential of artificial intelligence has also been recognized but the limitations and challenges of translating them into clinical practice along with their clinical applicability is an arguable point. The difficulty in understanding the AI curriculum can slow down the entire healthcare system and might be kept on the sidelines because of this inconvenience. Furthermore, the knowledge of artificial intelligence and training for the same has to be given to clinicians and healthcare providers. Recent advances in artificial intelligence present an exciting opportunity to improve healthcare; however, the translation of research techniques to effective clinical deployment present a new frontier for clinical and machines learning research.

A great sense of faith and responsibility has to be put in AI systems for them to be safe, applicable, and perform the best in the field of medicine. Artificial intelligence and machine learning surely bring in more utility, increased efficiency, and more productivity but only with right evaluation and implementation.

\section{CONCLUSION}

Medical data would only be increasing whether it be genome data or clinical data, but this can be very easily managed and processed using artificial intelligence and machine learning techniques. The future of the medical field is highly dependent on this data therefore processing this data using AI and ML techniques should be easy. What we require is to create a synergy between both systems for the success of the healthcare industry. There is a need to include learnings related to AI and ML in the curriculum of the future medical students so they are equipped to work with machine learning inclusive systems in order to make the best use of them.

The future needs of medicine can very well be predicted, diagnosed, and augmented by AI and machine learning. The use of these tools can help healthcare providers in doing the crux of their job rather than every facet of it. However, it is highly unlikely to see that AI will totally take over the field as there are certain limitations that still exist with implementation and learning of the same. The research paper has mentioned the advantages but to bring it in practicality will take time. A very balanced approach by healthcare providers towards artificial intelligence tools will surely bring a bright future to the field of medicine.

\section{REFERENCES}

1. Alam, MGR, Abedin, SF, Al Ameen, $M$ and Hong, CS (2016) Web of objects based ambient assisted living framework for emergency psychiatric state prediction. Sensors 16, 1431.

2. Frost \& Sullivan. 2016. From $\$ 600 \mathrm{M}$ to $\$ 6$ billion, artificial intelligence systems poised for dramatic market expansion in healthcare. https://ww2.frost.com/ news/ press-releases/ 600-m-6-billion-artificialintelligence-systems-poised-dramatic market-expansion-healthcare/.

3. Houssami N, Lee CI, Buist D, Tao D. 2017. Artificial intelligence for breast cancer screening: Opportunity or hype? The Breast 36(2017):31-33

4. King Jr B. 2018. Artificial intelligence and radiology: what will the future hold? Journal of the American College of Radiology 15(3 Part B):501503 DOI 0.1016/j.jacr.2017.11.017.

5. Kralj, K. and Kuka, M. (1998). "Using machine learning to analyze attributes in the diagnosis of coronary artery disease". In Proceedings of Intelligent Data Analysis in Medicine and PharmacologyIDAMAP98, Brighton, UK 
6. Lakhani P, Sundaram B. 2017. Deep learning at chest radiography: automated classification of pulmonary tuberculosis by using convolutional neural networks. Radiology

7. Meskó B. 2017. Artificial intelligence is the stethoscope of the 21st century. Available

at https://medicalfuturist.com/ibm-watson-is-thestethoscope-of-the-21st-century/

8. Norman A. 2018. Your future doctor may not be human. This is the rise of AI in medicine. Available at https://futurism.com/ aimedicine-doctor

9. Roach L. 2017. Artificial intelligence. Eyenet Magazine 77-83.

10. Samuel, A. L. (2000). "Some studies in machine learning using the game of checkers". IBM Journal of Research and Development. 44: 20626. doi: $10.1147 / \mathrm{rd} .441 .0206$. Pioneer of machine learning.

11. Zaidi D. 2018. The 3 most valuable applications of AI in healthcare. VentureBeat.

Available at https:// venturebeat.com/2018/ 04/ 22/the-3-most-valuable-applications

of-ai-in-health-carel

12. https://www.ncbi.nlm.nih.gov/pmc/articles/P $\underline{\mathrm{MC} 4345107 /}$

13. https://www.cambridge.org/core/journals/psy chological-medicine/article/machine-learning-inmental-health-a-scoping-review-of-methods-andapplications/0B70B1C827B3A4604C1C0102604 9F7D9

14. https://www.ncbi.nlm.nih.gov/pmc/articles/P MC5038709/

15.

https://www.ncbi.nlm.nih.gov/pmc/articles/PMC6 779111/ 